\title{
Spatial damping of compressional MHD waves in prominences
}

\author{
K. A. P. Singh ${ }^{1,2}$, B. N. Dwivedi ${ }^{1}$, and S. S. Hasan ${ }^{2}$ \\ 1 Department of Applied Physics, Institute of Technology, Banaras Hindu University, Varanasi-221005, India \\ e-mail: alkendra1978@yahoo.co.in; bholadwivedi@yahoo.com \\ 2 Indian Institute of Astrophysics, Bangalore 560034, India \\ e-mail: hasan@iiap.res.in
}

Received 26 September 2006 / Accepted 29 May 2007

ABSTRACT

\begin{abstract}
Aims. We study the spatial damping of linear compressional MHD waves in a homogeneous, isothermal, and unbounded prominence. Methods. We derive a general dispersion relation invoking the Newtonian radiation and turbulent viscosity. The turbulent viscosity is derived from SUMER and CDS observations for Kraichnan and Kolmogorov turbulences. Since we are interested in the spatial damping, the dispersion relation is solved numerically considering $\omega$ as real and $k$ as complex corresponding to slow, fast, and thermal modes.

Results. Both the slow and fast modes show strong damping, but the thermal mode is absent. The turbulent viscosity derived from observations can be a viable mechanism for the spatial damping of slow and fast modes. For a wave period of $1 \mathrm{~s}$, the damping length for slow and fast modes is found to be $1.1 \times 10^{2} \mathrm{~km}$ for the Kolmogorov turbulence. Correspondingly, the damping length of slow modes is $1.3 \times 10^{1} \mathrm{~km}$ and for fast modes $1.9 \times 10^{2} \mathrm{~km}$ for the Kraichnan turbulence. From the damping length study of slow modes, it is found that Kraichnan turbulence dominates for short wave periods between $10^{-7}$ to $10^{2} \mathrm{~s}$, and the Kolmogorov turbulence dominates for longer wave periods between $10^{3}$ to $10^{5} \mathrm{~s}$. From the damping length of fast modes, it is found that the Kraichnan turbulence dominates from very short to long wave periods.

Conclusions. The Kraichnan and Kolmogorov turbulence can be a viable damping mechanism for the spatial damping of short-period oscillations. In particular, the short-period oscillations $(5-15 \mathrm{~min})$ observed in quiescent limb prominences, which seem to be due to internal fundamental slow modes, have damping lengths in the range 1.9-3.7 $\times 10^{3} \mathrm{~km}$ for Kolmogorov turbulence and $3.5 \times 10^{3}-3.1 \times$ $10^{4} \mathrm{~km}$ for Kraichnan turbulence. Correspondingly, for fast modes, the damping length is in the range $2.6 \times 10^{5}-2.3 \times 10^{6} \mathrm{~km}$ for Kolmogorov turbulence and $1.7 \times 10^{7}-1.5 \times 10^{8} \mathrm{~km}$ for Kraichnan turbulence. This study underlines the importance of turbulent viscosity for explaining the damping of both slow and fast modes, which, hitherto, has not been explored.
\end{abstract}

Key words. Sun: atmosphere - Sun: prominences - Sun: oscillations

\section{Introduction}

Prominences are masses of relatively cool $\left(T \sim 10^{4} \mathrm{~K}\right)$ and dense $\left(\rho \sim 10^{-10} \mathrm{~kg} \mathrm{~m}^{-3}\right)$ material suspended in the corona $(\rho \sim$ $10^{-14} \mathrm{~kg} \mathrm{~m}^{-3}, T \sim 10^{6} \mathrm{~K}$ ). The spectra of prominences hold the key to understanding their physical conditions e.g., temperature, density, pressure, orientation, and strength of magnetic field. The internal structure and physical properties of prominences, however, can be studied best through prominence seismology.

Small amplitude waves (or oscillations) with velocity amplitudes from $0.1 \mathrm{~km} \mathrm{~s}^{-1}$ to $2-3 \mathrm{~km} \mathrm{~s}^{-1}$ have been observed (e.g., Bashkirtsev \& Mashnich 1984; Molowny-Horas et al. 1999). The analysis of time series of line width, line intensity, and Doppler velocity reveals the local nature of the oscillations, which have been classified, according to their periods as, short $(P<10 \mathrm{~min})$, intermediate $(10 \mathrm{~min}<P<40 \mathrm{~min})$, and longperiod oscillations $(P>40 \mathrm{~min})$, although very short periods of $30 \mathrm{~s}$ (Balthasar et al. 1993) and extreme ultra-long periods of more than $8 \mathrm{~h}$ (Foullon et al. 2004) have been reported. There have been few determinations of wavelength and phase speed for standing/propagating waves in prominences. Molowny-Horas et al. (1997) observed the oscillations in different parts of the quiescent prominence, and assuming the plane wave propagation, obtained the maximum values of phase speed $\left(\leq 44 \mathrm{~km} \mathrm{~s}^{-1}\right)$ and the perturbation wavelength $(\leq 20000 \mathrm{~km})$. Terradas et al. (2002) detected two propagating waves in a polar crown prominence, one with $\lambda \sim 67500 \mathrm{~km}, v_{\mathrm{ph}} \sim 15 \mathrm{~km} \mathrm{~s}^{-1}$ and the other with $\lambda \sim 50000 \mathrm{~km}, v_{\mathrm{ph}} \sim 12 \mathrm{~km} \mathrm{~s}^{-1}$, and found a standing wave with $\lambda \sim 44000 \mathrm{~km}, v_{\mathrm{ph}} \sim 12 \mathrm{~km} \mathrm{~s}^{-1}$.

There are several proofs of the damping of oscillations in prominences. Using the Vacuum Tower Telescope (VTT) at Sac Peak Observatory, Molowny-Horas et al. (1999) found velocity perturbations with periods between 28 and $95 \mathrm{~min}$ at different locations in a prominence. The amplitude of these oscillations was found to decrease in time with damping times between 101 and 377 min. Terradas et al. (2002) investigated the temporal and spatial variations of oscillations and reported a strong damping of oscillations with damping times between two to three times the wave period. On the theoretical side, Terradas et al. (2001) considered energy losses through Newtonian cooling to study the temporal damping of prominence oscillations and found that only slow mode waves are strongly damped, leaving fast mode waves almost undamped. Further explanations of the temporal damping of prominence oscillations have been offered by considering energy losses due to thermal conduction, optically thin radiation and heating (Carbonell et al. 2004; 
Terradas et al. 2005). However, a similar study of the spatial damping of prominence oscillations has not been done.

Temporal and spatial variations of prominence oscillations are the two different manifestations of the wave damping phenomenon. Observationally, damping time obtained from the decaying velocity perturbations are utilized (Molowny-Horas et al. 1999). Physical mechanisms such as radiative, viscous, electrical, or thermal losses are then invoked in the MHD equations. In spatial damping, however, one uses both indirect and direct methods, as well as the upper observed limit to the perturbation wavelength (Molowny-Horas et al. 1997) or the distance over which the amplitude of the wave is completely attenuated. For instance, Ballai (2003), in an order of magnitude estimation, studied the spatial damping of magnetoacoustic waves using the observed limit to the perturbation wavelength as the damping length. Recently, Carbonell et al. (2006) used the observations of Terradas et al. (2002) to show that the propagating wave amplitude decreased in a substantial way over a distance of $1-5 \times 10^{4} \mathrm{~km}$ for a wave period of 5 to $15 \mathrm{~min}$. Moreover, the thermal mode that arises due to thermal conduction is purely damped in the temporal study but propagating in the spatial damping study of prominence oscillations.

The damping mechanisms considered in earlier investigations (Ballai 2003; Terradas et al. 2001; Carbonell et al. 2004) only explain the damping of slow modes, so it is necessary to look for other viable damping mechanisms to explain the damping of fast mode waves. One of the possible candidates could be collisions between ions, electrons, and neutrals in partially ionized plasma. However, the damping of MHD waves by ionneutral interactions is much stronger for the fast-mode wave than for the slow-mode wave (Forteza et al. 2007). Given the poor knowledge of density and ionization fraction in prominences, it is hard to quantitatively estimate the role of partial ionization in the damping of fast-mode waves in prominences. The other possible candidate could be the wave damping due to the MHD turbulence that seems to be present in several emission lines of many different ionization states observed with SUMER and CDS. From these lines, the variation in non-thermal velocities with temperature in the range $2 \times 10^{4}$ to $7 \times 10^{5} \mathrm{~K}$ has been determined for the first time (Cirigliano et al. 2004). Similar variations in non-thermal velocities in CCTR and PCTR indicate that heating mechanisms in both could be the same with regard to wave propagation and MHD turbulence. De Boer et al. (1998), have calculated non-thermal velocities and found these to increase towards the periphery of the prominence. From temporal and spatial analysis, Terradas et al. (2002) observed damping of oscillations as a common feature in large areas, especially close to the edge of the prominence. Interpretation of non-thermal motions in terms of MHD turbulence is attractive because all nonthermal motions are isotropic and have very small scales that are often displayed on the basis of their observational characteristics and physical conditions in prominence, PCTR, CCTR, and the solar corona. Inhomogeneous structures caused by density stratification and magnetic field may also play a role in driving turbulence (Chae et al. 1998). The presence of non-thermal velocities and damping of magnetoacoustic waves can be explained by MHD turbulence, so the compelling body of observational evidence points towards the MHD turbulence for the likely explanation of both slow and fast-mode waves. In this paper, we derive a general dispersion relation invoking the Newtonian radiation and turbulent viscosity. The turbulent viscosity is derived from the observations of SUMER and CDS for Kraichnan and Kolmogorov turbulences in Sect. 2. We present the results and discussion in Sect. 3 and conclusions in the last section.

\section{General dispersion relation}

We consider a homogeneous equilibrium configuration with a magnetic field in the $x$-direction. Neglecting the effect of gravity, we have

$p_{0}=$ const., $\quad \rho_{0}=$ const.,

$T_{0}=$ const., $\quad \boldsymbol{B}_{0}=B_{0} \boldsymbol{x}, \quad$ and $\boldsymbol{V}_{0}=0$,

where $p_{0}, \rho_{0}, T_{0}$, and $B_{0}$ are the equilibrium values of the pressure, density, temperature and magnetic field.

The basic equations for the discussion of linear MHD waves are

$\frac{\mathrm{D} \rho}{\mathrm{D} t}+\rho \boldsymbol{\nabla} \cdot \boldsymbol{V}=0$

$\rho \frac{\mathrm{D} \boldsymbol{V}}{\mathrm{D} t}=-\nabla p+\frac{1}{\mu}(\nabla \times \boldsymbol{B}) \times \boldsymbol{B}+\rho \eta_{\mathrm{t}} \nabla^{2} \boldsymbol{V}$

$\frac{\rho^{\gamma}}{\gamma-1} \frac{\mathrm{D}}{\mathrm{D} t}\left(\frac{p}{\rho^{\gamma}}\right)=Q_{\text {viscous }}-Q_{\text {radiation }}$

$\frac{\partial \boldsymbol{B}}{\partial t}=\nabla \times(\boldsymbol{V} \times \boldsymbol{B})$

$\nabla \cdot \boldsymbol{B}=0$

$p=\rho R T$

where $\eta_{\mathrm{t}}\left(=v_{\mathrm{obs}} \delta\right)$ is the coefficient of turbulent kinematic viscosity and $\gamma$ the ratio of specific heats. Also, $v_{\text {obs }}$ is the turbulent velocity from SUMER and CDS observations (Cirigliano et al. 2004) and $\delta$ is a typical turbulence length-scale in prominences that has been inferred to be $15 \mathrm{~km}$ for Kraichnan (Kraichnan 1965) and $1000 \mathrm{~km}$ for Kolmogorov turbulence (Kolmogorov 1941). The Newtonian radiation has been invoked by means of the simplified energy equation $\frac{\rho^{\hat{\gamma}}}{\hat{\gamma}-1} \frac{\mathrm{D}}{\mathrm{D} t}\left(\frac{P}{\rho^{\gamma}}\right)=0$, where $\hat{\gamma}$ is given by $\hat{\gamma}=\frac{1+i \omega \tau_{\mathrm{R}} \gamma}{1+\mathrm{i} \omega \tau_{\mathrm{R}}}$ (Bunte $\&$ Bogdan 1994) and $\tau_{R}$ is the radiative relaxation time. The MHD equations are linearized and perturbations of the form $\operatorname{expi}(\omega t+\boldsymbol{k} \cdot \boldsymbol{r})$ are taken where $\omega$ is the frequency of oscillations and $\boldsymbol{k}$ the wave number. Using $x=l \bar{x}, \bar{k}=l k, V=C_{\mathrm{s}} \bar{V}, \omega=\frac{C_{\mathrm{s}}}{l} \bar{\omega}, B=B_{0} \bar{B}_{x}, p=\frac{B_{0}^{2}}{\mu} \bar{p}$, $\rho=\frac{\gamma B_{0}^{2} \bar{\rho}}{\mu C_{\mathrm{s}}^{2}}$, and $\eta_{\mathrm{t}}=\bar{\eta}_{\mathrm{t}} C_{\mathrm{s}} l$, equations are cast into the dimensionless form where $l(=5236 \mathrm{~km})$ is a "dimensionless length" and the quantities with a bar on top denote non-dimensional quantities. The resulting general dispersion relation is

$a_{4} k^{4}+a_{2} k^{2}+a_{0}=0$,

where the coefficients are given by

$a_{0}=\omega^{5}-\mathrm{i} \frac{1}{\tau_{\mathrm{R}}} \omega^{4}$

$$
\begin{aligned}
a_{2}= & -2 \mathrm{i} \eta_{\mathrm{t}} \omega^{4}-\left(1+V_{\mathrm{A}}^{2}+2 \frac{\eta_{\mathrm{t}}}{\tau_{\mathrm{R}}}\right) \omega^{3} \\
& +\mathrm{i} \frac{1}{\gamma \tau_{\mathrm{R}}} \omega^{2}+\mathrm{i} \frac{V_{\mathrm{A}}^{2}}{\tau_{\mathrm{R}}} \omega^{2}
\end{aligned}
$$

and

$$
\begin{aligned}
a_{4}= & -\eta_{\mathrm{t}}^{2} \omega^{3}+\mathrm{i} \eta_{\mathrm{t}} \omega^{2}+\mathrm{i} \eta_{\mathrm{t}} V_{\mathrm{A}}^{2} \omega^{2}+\mathrm{i} \frac{\eta_{\mathrm{t}}^{2}}{\tau_{\mathrm{R}}} \omega^{2}+\frac{\eta_{\mathrm{t}}}{\gamma \tau_{\mathrm{R}}} \omega \\
& +\frac{\eta_{\mathrm{t}} V_{\mathrm{A}}^{2}}{\tau_{\mathrm{R}}} \omega+\cos ^{2} \theta V_{\mathrm{A}}^{2} \omega-\mathrm{i} \frac{\cos ^{2} \theta V_{\mathrm{A}}^{2}}{\gamma \tau_{\mathrm{R}}}
\end{aligned}
$$



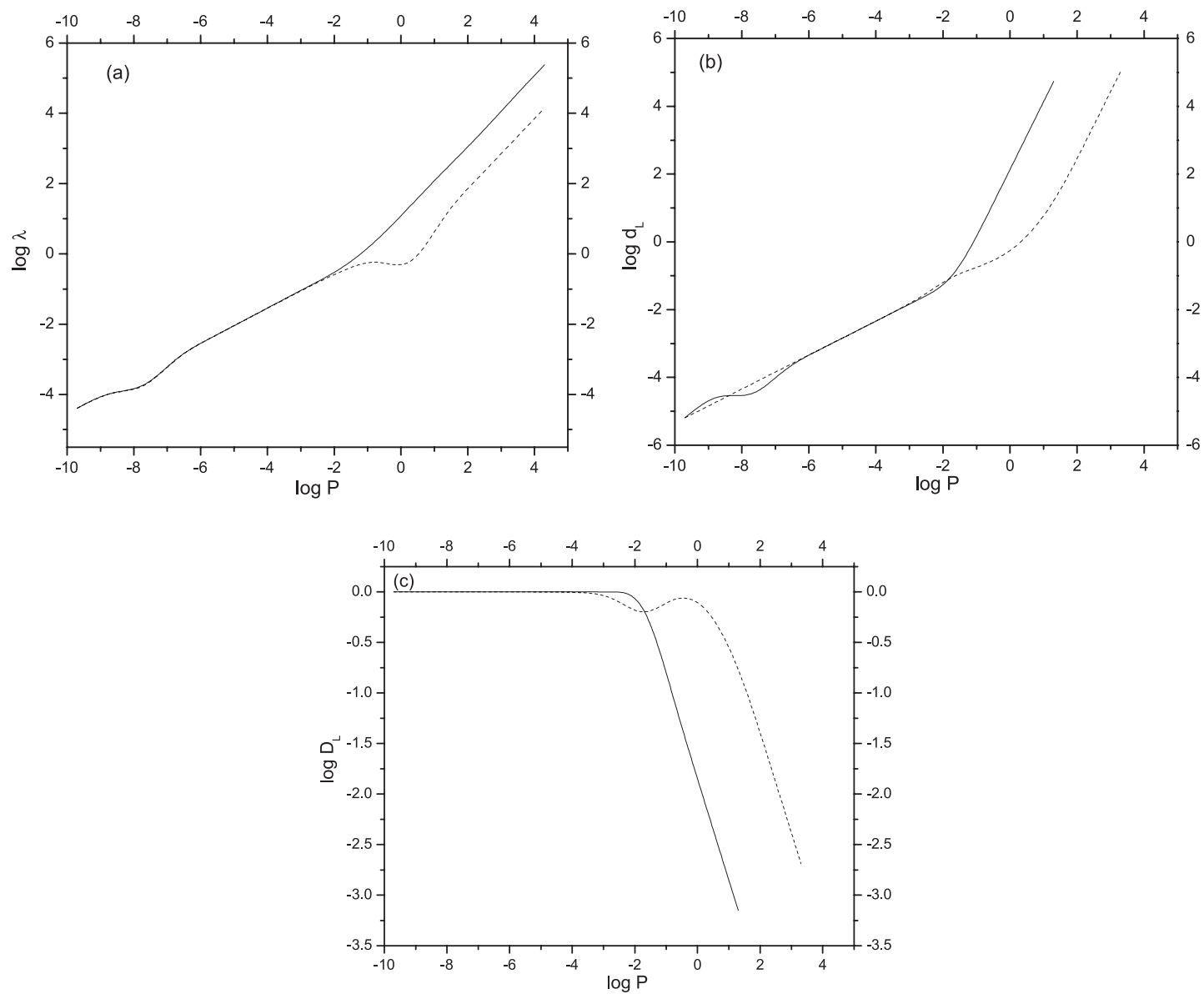

Fig. 1. a) Wavelength b) damping length and c) damping per wavelength as a function of wave period for slow (shown by a dotted line) and fast mode wave (shown by a solid line) for Kolmogorov turbulence.

In case of turbulent viscosity alone, the dispersion relation has the similar mathematical form as Eq. (8) with

$a_{0}^{\prime}=\omega^{5}$,

$a_{2}^{\prime}=-2 \mathrm{i} \eta_{\mathrm{t}} \omega^{2}-\left(1+V_{\mathrm{A}}^{2}\right) \omega^{3}$, and

$a_{4}^{\prime}=-\eta_{\mathrm{t}}^{2} \omega^{3}+\mathrm{i} \eta_{\mathrm{t}} \omega^{2}+\mathrm{i} \eta_{\mathrm{t}} V_{\mathrm{A}}^{2} \omega^{2}+\cos ^{2} \theta V_{\mathrm{A}}^{2} \omega$

with $k_{x}=k \cos \theta$ and $k=\sqrt{k_{x}^{2}+k_{z}^{2}}$.

Since we are interested in the spatial damping of magnetoacoustic waves, we consider the frequency, $\omega$, to be real and seek complex solutions of the wavenumber $k$ expressed as $k=k_{\mathrm{r}}+\mathrm{i} k_{\mathrm{i}}$. Equation (8) is a fourth-order polynomial for $k$ as a function of $\omega$, and from it we can obtain four complex roots corresponding to slow and fast modes, but thermal mode is absent. In principle, an important difference in the spatial damping case with respect to temporal damping is that the thermal wave is a propagating wave instead of a simple decaying oscillation. The wavelength of the waves is given by $\lambda=\frac{2 \pi}{k_{\mathrm{r}}}$, the damping length by $d_{\mathrm{L}}=\frac{1}{k_{\mathrm{i}}}$, and the damping per wavelength is $D_{\mathrm{L}}=\frac{k_{\mathrm{i}}}{k_{\mathrm{r}}}$, which we have explored using typical values of the density, temperature, and magnetic field for prominences from Carbonell et al. (2006).

\section{Results and discussion}

The dispersion relation (8) has been solved numerically for a magnetic field of $10 \mathrm{G}$ and a propagation angle of $\pi / 4$. Before considering the range of periods typical of the detected oscillations in prominences, we solved Eq. (8) for prominence regime 1.1 (Carbonell et al. 2006), considering a period ranging from very short $\left(10^{-7} \mathrm{~s}\right)$ to very long $\left(10^{7} \mathrm{~s}\right)$. The behavior of the wavelength, damping length, and damping per wavelength as a function of wave period is shown in Figs. 1a-c. Figure 1a shows the wavelength of slow and fast modes as a function of the wave period. Figure $1 \mathrm{~b}$ shows that the damping length of slow and fast modes is almost the same for short wave periods, while Fig. 1c shows that both the slow and fast modes are strongly damped for shorter wave periods. We also see that the fast modes propagate undamped at higher wave periods $\left(10^{5}-10^{7} \mathrm{~s}\right)$.

We then consider the behavior of damping lengths of magnetoacoustic waves as a function of wave period for various cases. In Fig. 2a, we compare the damping length of slow modes as a function of wave period for Kraichnan and Kolmogorov turbulences. This shows that for short wave periods between $10^{-7}$ to $10^{2} \mathrm{~s}$, Kraichnan turbulence dominates because the damping length for Kraichnan turbulence is shorter than the Kolmogorov turbulence. However, Kolmogorov turbulence dominates for long wave periods $\left(10^{3}-10^{5} \mathrm{~s}\right)$. Figure $2 \mathrm{~b}$ shows the damping length of fast modes as a function of wave period for Kraichnan and Kolmogorov turbulence, and it is the Kraichnan turbulence that dominates from very short to long periods. In Fig. 3a, we compare the damping length of slow modes obtained due to Newtonian cooling and due to Kolmogorov turbulence. This shows that the Kolmogorov turbulence strongly dominates for short wave periods and approaches the Newtonian cooling case 

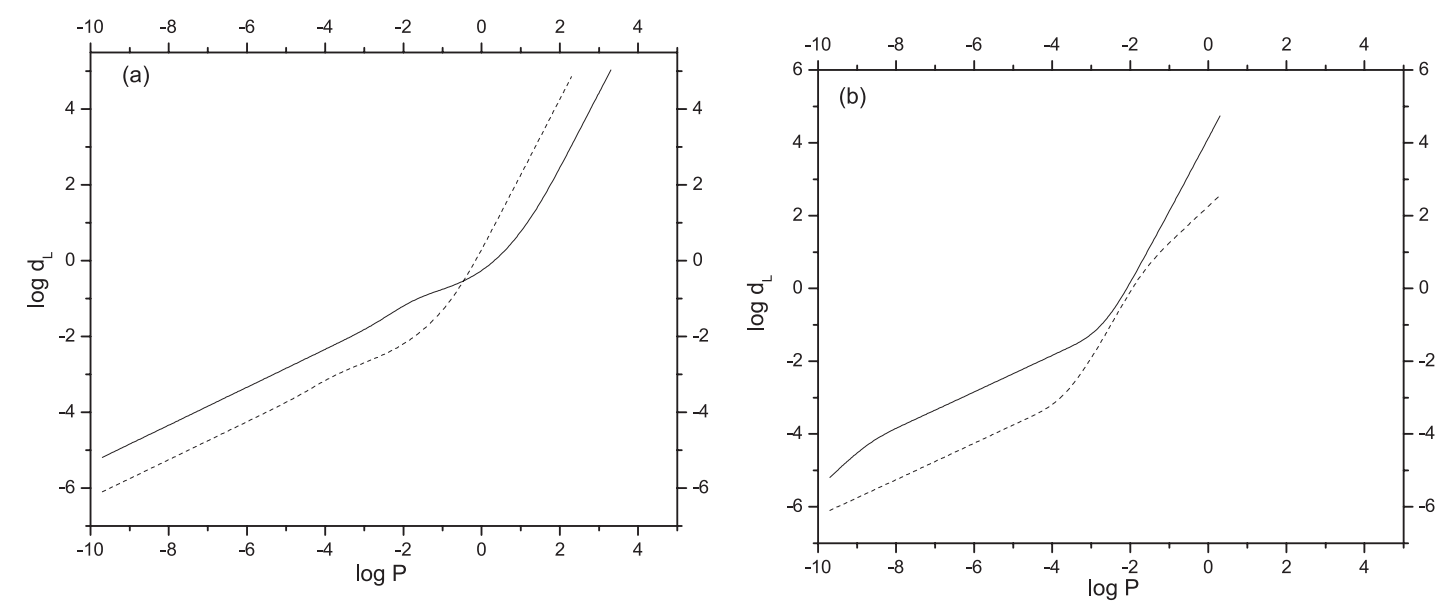

Fig. 2. Damping length for Kraichnan (shown by a dashed line) and Kolmogorov (shown by a solid line) turbulences as a function of wave period for a) slow mode and b) fast mode.
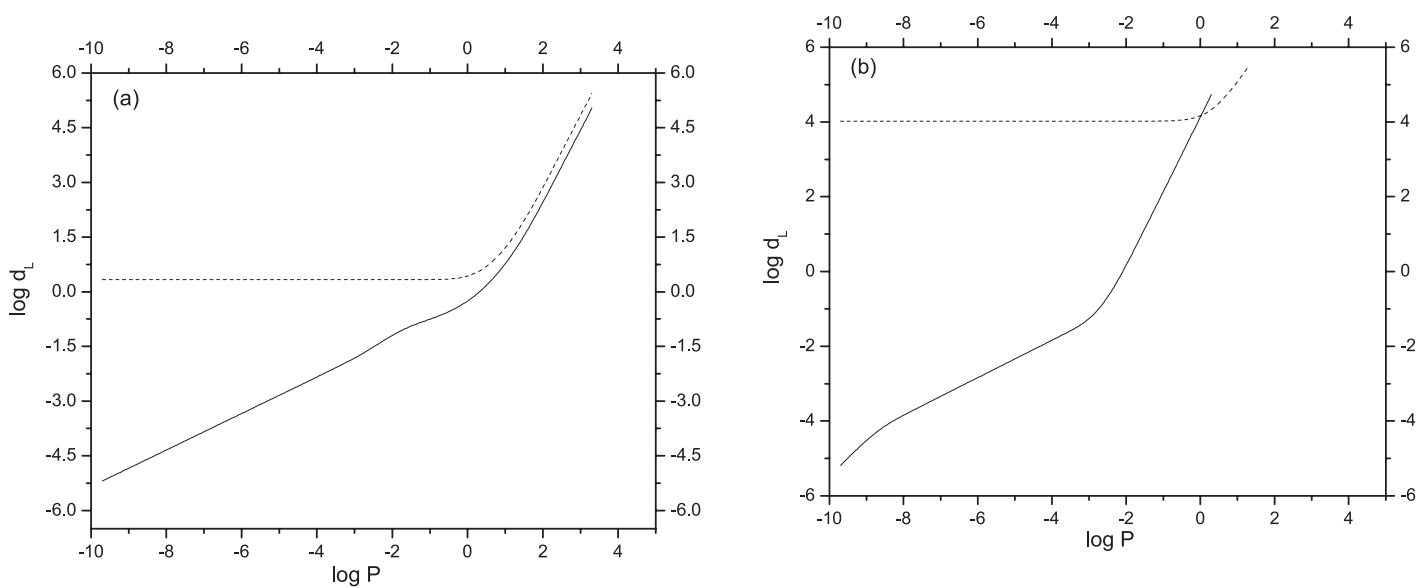

Fig. 3. Damping length for Newtonian radiation (shown by a dashed line) and Kolmogorov turbulences (shown by a solid line) as a function of wave period for a) slow mode and $\mathbf{b}$ ) fast mode.

for long wave periods. As shown in Fig. 3b, the Kolmogorov turbulence also dominates for the fast modes.

We have modified the values of the physical parameters, such as magnetic field, temperature, density, and the propagation angle in an attempt to show their dependence on the damping length of slow and fast modes. Figure 4 shows the variation in the damping length for different values of magnetic field strength. As shown in Fig. 4a, the damping length of slow modes remains unaffected by the variation of magnetic field strength. However, for fast modes, the damping length increases with the increase in the magnetic field strength at longer wave periods but remains unaffected at shorter wave periods (Fig. 4b). The variation in damping length for different values of the prominence temperature is shown in the Fig. 5. The damping length of slow modes remains unaffected at shorter wave periods but increases at longer wave periods, as shown in Fig. 5a, and the fast modes remain unaffected (Fig. 5b). In Fig. 6 the damping length for different values of prominence density is shown. Figure 6 a shows that the damping length of slow modes remains unaffected by an increase in density, whereas it decreases for fast modes (Fig. 6b). The variation in damping length for different values of propagation angle are shown in Figs. 7a, b. This shows that the damping length of slow and fast modes remains unaffected at shorter wave periods but decreases at longer wave periods with increase in propagation angle.

\section{Conclusions}

In this paper, we have studied the spatial damping of linear magnetoacoustic waves by deriving a general dispersion relation invoking the Newtonian radiation and turbulent viscosity for Kraichnan and Kolmogorov turbulences that is derived from the SUMER and CDS observations of Cirigliano et al. (2004). The dispersion relation has been numerically solved to study the damping length of slow and fast modes.

The main conclusions that can be extracted from our study are:

1. Slow and fast modes are strongly damped by turbulence but the thermal mode is absent.

2. From the damping length study of slow modes, it is found that Kraichnan turbulence dominates for short wave periods between $10^{-7}$ to $10^{2} \mathrm{~s}$ during which time Kolmogorov turbulence dominates for longer wave periods $\left(10^{3}-10^{5} \mathrm{~s}\right)$.

3. From the damping length of fast modes, it is found that the Kraichnan turbulence dominates from very short to long wave periods.

It is now interesting to compare the theoretical damping length with the value derived from the observations. In Terradas et al. (2002), the dominant period of the oscillations, interpreted as slow modes, was around $75 \mathrm{~min}$, and correspondingly the damping length is found to be $1.7 \times 10^{4} \mathrm{~km}$ for Kolmogorov 

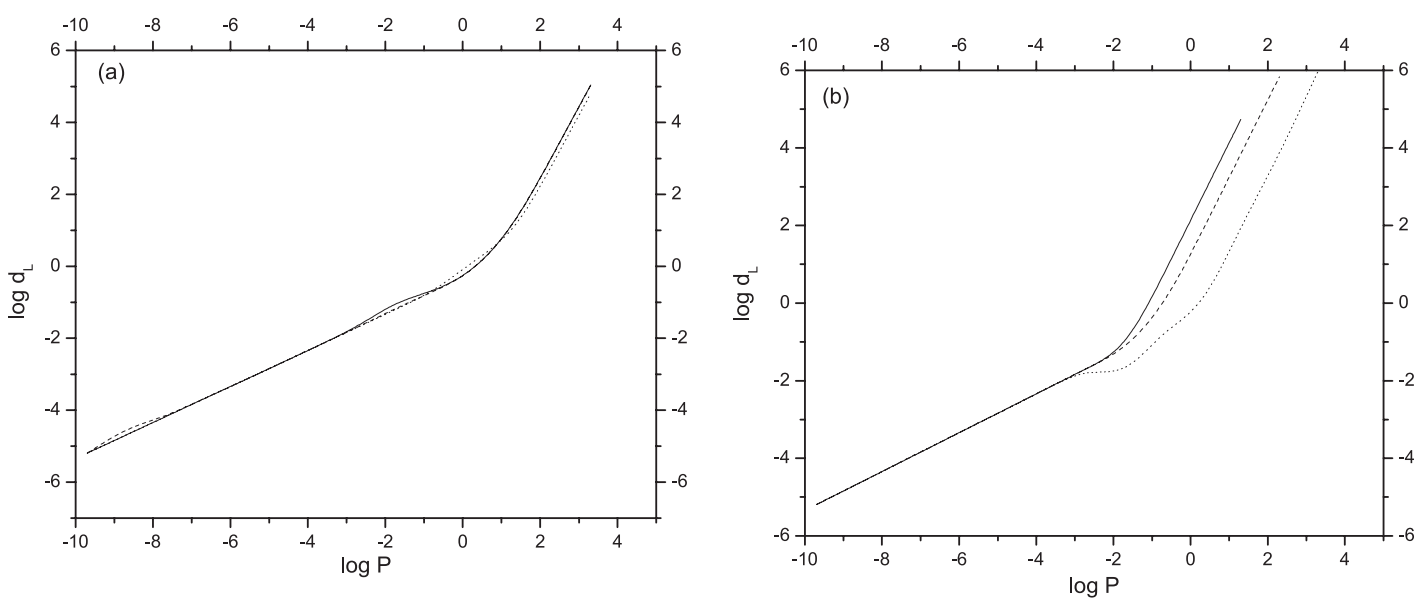

Fig. 4. Damping length for $B=1 \mathrm{G}$ (shown by a dotted line), $B=5 \mathrm{G}$ (shown by a dashed line), and $B=10 \mathrm{G}$ (shown by a solid line) as a function of wave period for $\mathbf{a}$ ) slow mode and $\mathbf{b}$ ) fast mode.
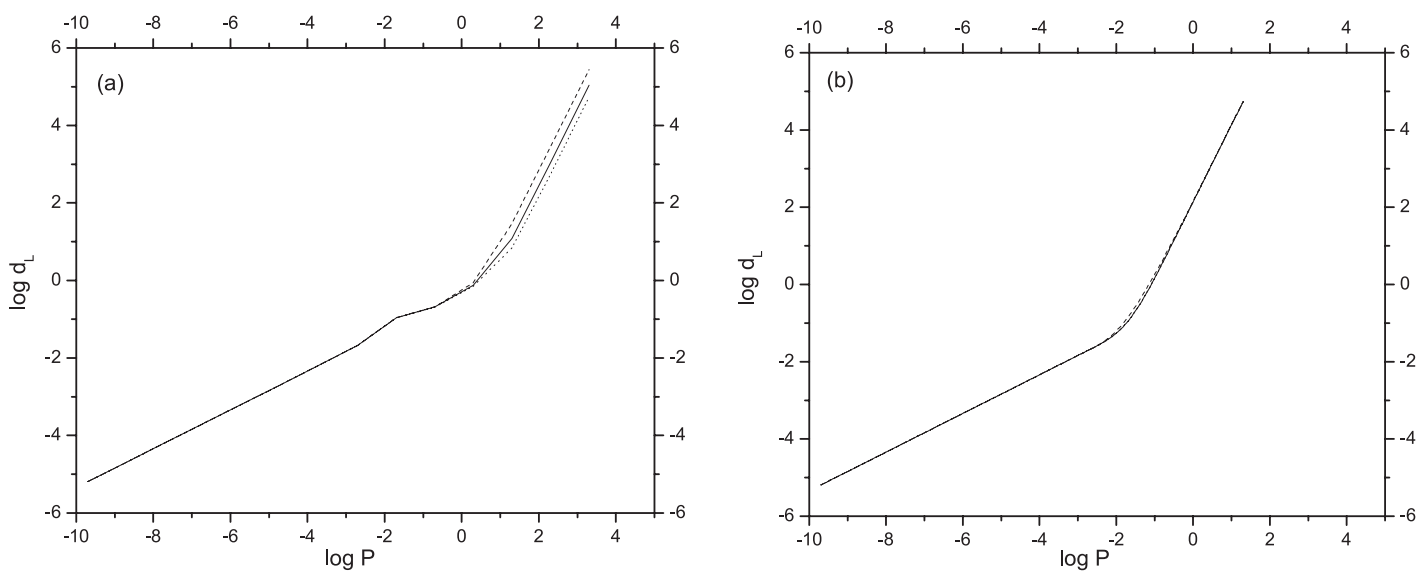

Fig. 5. Damping length for $T=5000 \mathrm{~K}$ (shown by a dotted line), $T=15000 \mathrm{~K}$ (shown by a dashed line), and $T=8000 \mathrm{~K}$ (shown by a solid line) as a function of wave period for $\mathbf{a})$ slow mode and $\mathbf{b}$ ) fast mode.
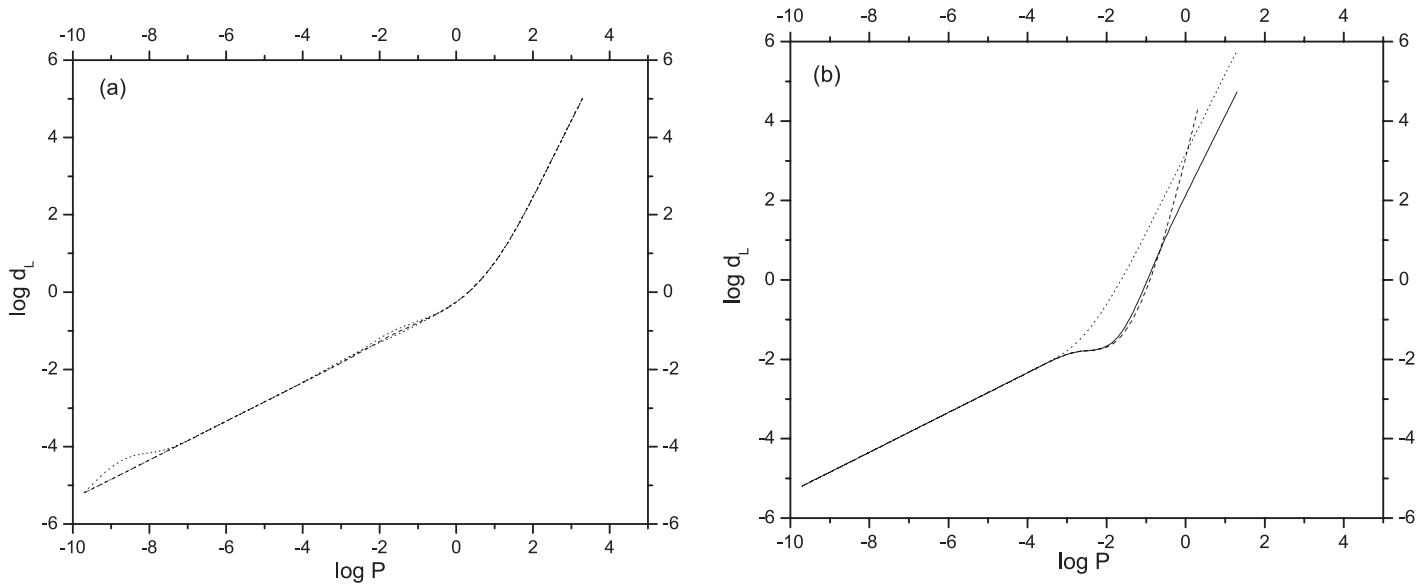

Fig. 6. Damping length for $\rho=1 \times 10^{-11} \mathrm{~kg} \mathrm{~m}^{-3}$ (shown by a dotted line), $\rho=10 \times 10^{-11} \mathrm{~kg} \mathrm{~m}^{-3}$ (shown by a dashed line), and $\rho=5 \times 10^{-11} \mathrm{~kg} \mathrm{~m}{ }^{-3}$ (shown by a solid line) as a function of wave period for $\mathbf{a}$ ) slow mode and $\mathbf{b}$ ) fast mode.

turbulence and $7.1 \times 10^{5} \mathrm{~km}$ for the Kraichnan turbulence. For fast modes, the damping length is $5.8 \times 10^{7} \mathrm{~km}$ for Kolmogorov turbulence and $3.8 \times 10^{9} \mathrm{~km}$ for Kraichnan turbulence. For the wave periods between $5 \mathrm{~min}$ and $15 \mathrm{~min}$, the damping length corresponding to slow mode is $1.9-3.7 \times 10^{3} \mathrm{~km}$ for Kolmogorov turbulence and $3.5 \times 10^{3}-3.1 \times 10^{4} \mathrm{~km}$ for Kraichnan turbulence. Correspondingly, for fast modes the damping length is in the range $2.6 \times 10^{5}-2.3 \times 10^{6} \mathrm{~km}$ for Kolmogorov turbulence and $1.7 \times 10^{7}-1.5 \times 10^{8} \mathrm{~km}$ for the Kraichnan turbulence. For a wave period of $1 \mathrm{~s}$, the damping length for slow, and the fast mode is found to be $1.1 \times 10^{2} \mathrm{~km}$ for Kolmogorov turbulence. Correspondingly, the damping length of slow modes is $1.3 \times 10^{1} \mathrm{~km}$ and of fast modes is $1.9 \times 10^{2} \mathrm{~km}$ for the Kraichnan turbulence.

In summary, we underline the importance of turbulence on the spatial damping of prominence oscillations for both slow and 

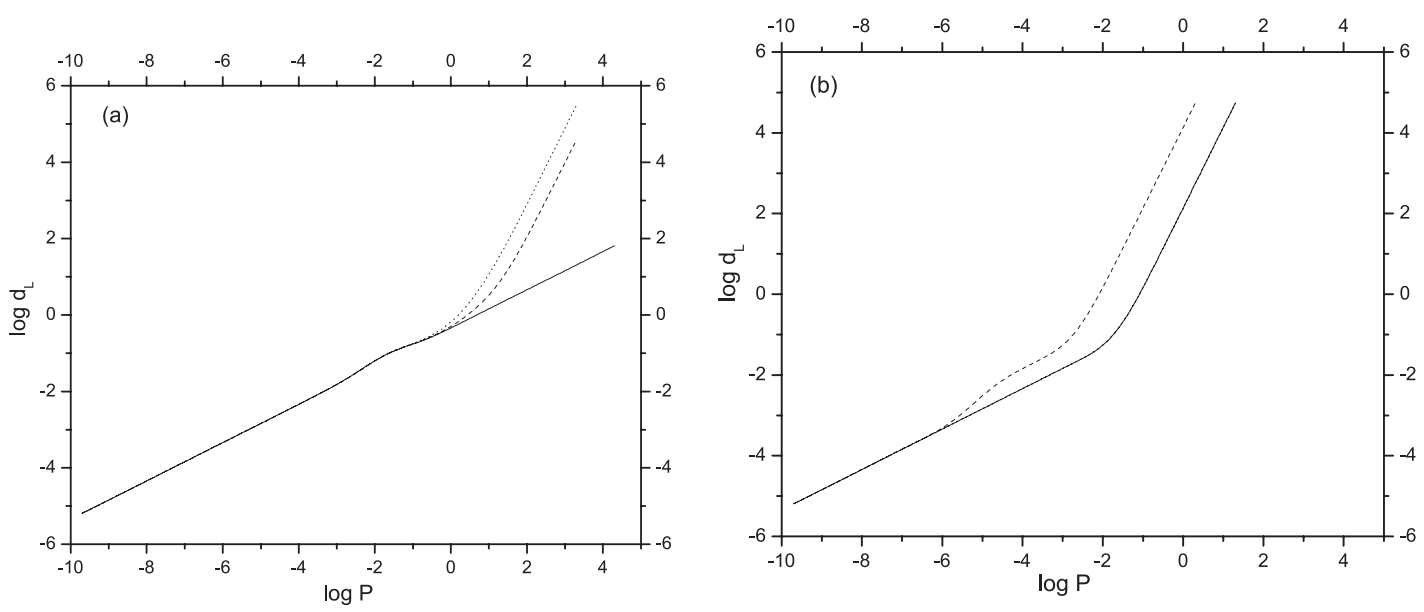

Fig. 7. Damping length for $\theta=10^{-2}$ (shown by a dotted line), $\theta=\pi / 3$ (shown by dashed line), and $\theta=\pi / 2$ (shown by a solid line) as a function of wave period for a) slow mode and $\mathbf{b}$ ) fast mode.

fast mode waves. The previous investigations either explain the damping of slow or fast modes in prominences. The inclusion of turbulence explains the damping of both slow and fast modes. This is a novel element based on a compelling body of evidence from the SUMER and CDS observations for the cases of Kraichnan and Kolmogorov turbulence. New observations from the Hinode spacecraft may shed new light on the turbulence prevalent in all the solar structures.

Acknowledgements. KAPS acknowledges the University Grants Commission, New Delhi, for the award of a senior research fellowship. We express our gratitude to Valery M. Nakariakov and the referee for their valuable comments, which improved the manuscript.

\section{References}

Ballai, I. 2003, A\&A, 410, L17

Balthasar, H., Wiehr, E., Schleicher, H., \& Wohl, H. 1993, A\&A, 277, 635
Bashkirtsev, V. S., \& Mashnich, G. P. 1984, Sol. Phys., 91, 93 De Boer, C. R., Stellmacher, G., \& Wiehr, E. 1998, A\&A, 334, 280 Bunte, M., \& Bogdan, T. J. 1994, A\&A, 283, 642

Carbonell, M., Oliver, R., \& Ballester, J. L. 2004, A\&A, 415, 739

Carbonell, M., Terradas, J., Oliver, R., \& Ballester, J. L. 2006, A\&A, 460, 573

Chae, J., Schühle, U., \& Lemaire, P. 1998, ApJ, 505, 957

Cirigliano, D., Vial, J. C., \& Rovira, H. 2004, Sol. Phys., 223, 95

Forteza, P., Oliver, R., Ballester, J. L., \& Khodachenko, M. L. 2007, A\&A, 461, 731

Foullon, C., Verwichte, E., \& Nakariakov, V. M. 2004, A\&A, 427, 25

Kolmogorov, A. N. 1941, C. R. Acad. Sci. URSS, 30, 301

Kraichnan, R. H. 1965, Phys. Fluids, 8, 1385

Molowny-Horas, R., Oliver, R., Ballester, J. L., \& Baudin, F. 1997, Sol. Phys., 172,181

Molowny-Horas, R., Wiehr, E., Balthasar, H., Oliver, R., \& Ballester, J. L. 1999, in JOSO Annual Report 1998, ed. A. Antalová, H. Balthasar, \& A. Kuèera, Astronomical Institute Tatranská Lomnica, 126

Terradas, J., Oliver, R., \& Ballester, J. L. 2001, A\&A, 378, 635

Terradas, J., Molowny-Horas, R., Wiehr, E., et al. 2002, A\&A, 393, 637

Terradas, J., Carbonell, M., Oliver, R., \& Ballester, J. L. 2005, A\&A, 434, 741 